\title{
OPEN An efficient simulation for quantum secure multiparty computation
}

\begin{abstract}
Kartick Sutradhar ${ }^{\bowtie}$ \& Hari Om
The quantum secure multiparty computation is one of the important properties of secure quantum communication. In this paper, we propose a quantum secure multiparty summation (QSMS) protocol based on $(t, n)$ threshold approach, which can be used in many complex quantum operations. To make this protocol secure and realistic, we combine both the classical and quantum phenomena. The existing protocols have some security and efficiency issues because they use $(n, n)$ threshold approach, where all the honest players need to perform the quantum multiparty summation protocol. We however use a $(t, n)$ threshold approach, where only $t$ honest players need to compute the quantum summation protocol. Compared to other protocols our proposed protocol is more cost-effective, realistic, and secure. We also simulate it using the IBM corporation's online quantum computer, or quantum experience.
\end{abstract}

In quantum internet, the secure quantum communication is an essential property. The secure quantum communication can be provided by the quantum key distribution $(\mathrm{QKD})^{1-5}$, secure quantum channel ${ }^{6-9}$, dense quantum measurement ${ }^{10-13}$, and quantum secure multiparty summation (QSMS). In quantum computing, the QSMS is a fundamental paradigm for secure quantum communication ${ }^{14-19}$. The QSMS can be used to build many complex protocols ${ }^{20-31}$ like multiplication, sorting ${ }^{32-35}$, voting ${ }^{36,37}$, auction, etc. The QSMS includes a list of secrets $\mathbb{S}$ and a set of players $\mathbb{P}$. The list of secrets is shared among $n$ players $\mathbb{P}=\left\{P_{1}, P_{2}, \ldots, P_{n}\right\}$ over a finite field $\mathbb{F}_{d}$, where $d$ denotes a large prime. The players $\mathbb{P}=\left\{P_{1}, P_{2}, \ldots, P_{n}\right\}$ jointly perform the summation by without disclosing the privacy of their secrets. The security of this protocol is guaranteed until some players reveal their secrets. Suppose, the dealers $A$ and $B$ contain two secrets $X$ and $Y$ (for simplicity, we take only two secrets, but the secrets can be any number $n$ or more than $n$ ) and the players $\mathbb{P}=\left\{P_{1}, P_{2}, \ldots, P_{n}\right\}$ want to compute the secure summation without revealing their secrets. Consider that $X, Y \in \mathbb{S}$ are two secrets of the dealers $A$ and $B$, respectively. The dealers $A$ and $B$ share two secrets $X$ and $Y$ among $n$ players $\mathbb{P}=\left\{P_{1}, P_{2}, \ldots, P_{n}\right\}$ using the Shamir's secret sharing ${ }^{38}$. The $X_{1}, X_{2}, \ldots, X_{n}$ and $Y_{1}, Y_{2}, \ldots, Y_{n}$ denote the shares of secrets $X$ and $Y$, respectively. The players $\mathbb{P}=\left\{P_{1}, P_{2}, \ldots, P_{n}\right\}$ want to execute $\left(X_{i}+Y_{i}\right), i=1,2, \ldots, n$, without disclosing their shares. We simulate this protocol by using the IBM quantum computer or quantum experience ${ }^{39,40}$, which is presented at T.J. Watson lab in USA. The novelties of this QSMS can be summarized as follows.

- The proposed protocol is more secure against the participant attack and it has the threshold approach of $(t, n)$, in which only the $t$ honest players can securely compute the multiparty quantum summation.

- Compared to other protocols, the proposed protocol is more realistic and cost-effective.

In secure multiparty classical computation, there exist many summation protocols, but they are unable to provide secure communications; whereas, the QSMS is unconditional secure as it uses the concepts of quantum mechanics. In secure multiparty quantum computation, there have been discussed many summation protocols. In 2002, Heinrich ${ }^{41}$ discussed a QSMS protocol. In 2003, Heinrich ${ }^{42}$ introduced another QSMS protocol with Boolean setting. In 2006, Hillery ${ }^{43}$ discussed a QSMS, based on two-particle entanglement. In 2007, Du et al. ${ }^{44}$ discussed a QSMS protocol based on non-orthogonal states. This protocol's modulo is $n+1$, where total number of players is $n$. In 2010, Chen et al. ${ }^{45}$ implemented a QSMS protocol based on multi-particle entanglement with modulo 2. In 2014, Zhang et al. ${ }^{46}$ discussed a QSMS protocol based on polarization of photon with modulo 2. In 2015, Zhang et al. ${ }^{47}$ implemented a quantum summation protocol for three-party with modulo 2 . There are some limitations in the above mentioned protocols ${ }^{44-47}$, as discussed below.

- These protocols are based on a threshold approach of $(n, n)$, where all players need to perform the secure multiparty quantum summation. If any player is rational, then these protocols cannot be executed efficiently. 
- These protocols are not cost-efficient because they have bit-by-bit operations.

- These protocols have some security issues because their modulo is too small.

Shi et al. ${ }^{48}$ implemented a QSMS protocol, which can compute the summation efficiently with large modulo $p$, but it has the threshold approach of $(n, n)$, where $p=2^{q}$ and $q$ is number of qubits. Shi and Zhang ${ }^{49}$ discussed a QSMS protocol, which can compute the summation efficiently, but it is not secure because it has only two-party. Zhang et al..$^{50}$ implemented a QSMS protocol based on quantum secure multiparty computation, but its modulo is 2 only. Liu et al. ${ }^{51}$ discussed a QSMS protocol based on the threshold approach of $(n, n)$ with modulo 2 , and its form of computation is bit-by-bit. In 2018, Yang and Ye ${ }^{52}$ discussed a QSMS protocol with modulo $d$. Its form of computation is secret-by-secret, but it has the threshold approach of $(n, n)$. In 2019, Jiao et al. ${ }^{53}$ discussed a QSMS protocol, which has the threshold approach of $(n, n)$, and its form of computation is bit-by-bit. In the same year, Zhang et al. ${ }^{54}$ have discussed a QSMS protocol. Its modulo is $d$, but it has the threshold approach of $(n, n)$. In 2020, Sutradhar and Om introduced a quantum secret sharing ${ }^{55}$ protocol. This protocol is efficient and has $(t, n)$ threshold approach, but it has more computational cost because it uses CNOT gate and SHA1. This protocol does not discuss about the realistic implementation, collective and coherent attacks. In the same year, Sutradhar and $\mathrm{Om}^{56}$ discussed a multiparty quantum summation protocol. This protocol is efficient and has $(k, n)$ threshold approach, but it has more computational cost because it uses SUM gate, where $k$ denotes the players of the qualified subset. This protocol does discuss about the collective and coherent attacks. Recently, Sutradhar and $\mathrm{Om}^{57}$ introduced another quantum protocol for secure multiparty summation. This protocol is efficient and has $(t, n)$ threshold approach, but it has more computational cost because it uses the SUM gate. This protocol does not discuss about the realistic implementation. Moreover, the proposed protocol is more secure, realistic and cost-effective as compared to the these protocols ${ }^{55-57}$. In this paper, we propose a QSMS protocol with a form of secret-by-secret computation. The proposed protocol has the threshold approach of $(t, n)$, where only $t$ honest players need to execute the secure multiparty quantum summation efficiently and cost-effectively without disclosing their secrets.

\section{Preliminaries}

In this section, we discuss the Shamir's Secret Sharing (SSS), Pauli operator, and Quantum Fourier Transform $(Q F T)$.

Shamir's secret sharing. The $\operatorname{SSS}^{38}$ contains $\mathbb{P}=\left\{P_{1}, P_{2}, \ldots, P_{n}\right\}$, a dealer, and $n$ players. It is formed in two phases as discussed below.

Secret sharing phase. In this phase, the dealer uses $(t-1)$-degree polynomial $f(x)$ to share the secret and distribute those shares among $n$ players, each player $P_{i}$ contains only $f\left(x_{i}\right), i=1,2, \ldots, n$.

Secret reconstruction phase. In this phase, reconstruction is performed by the threshold number of players using the Lagrange Interpolation, as discussed below.

$$
f(x)=\sum_{u=1}^{t} f\left(x_{u}\right) \prod_{1 \leq z \leq t, z \neq u} \frac{x-x_{z}}{x_{u}-x_{z}}
$$

For $x=0$, Eq. (1) can be simplified as follows:

$$
\begin{aligned}
f(0) & =\sum_{u=1}^{t} f\left(x_{u}\right) \prod_{1 \leq z \leq t, z \neq u} \frac{-x_{z}}{x_{u}-x_{z}} \\
& =\sum_{u=1}^{t} f\left(x_{u}\right) \prod_{1 \leq z \leq t, z \neq u} \frac{x_{z}}{x_{z}-x_{u}}
\end{aligned}
$$

where $u, z=1,2, \ldots, t$.

Pauli operator. The Pauli operator is defined as follows:

$$
U_{m, 0}=\sum_{c=0}^{d-1} \omega^{c .0}|c+m\rangle\langle c|
$$

where $m \in\{0,1, \ldots, d-1\}$.

Quantum Fourier transform (QFT). The $Q F T^{58}$ is an extension of the regular Fourier discrete transformation. For $v \in\{0,1, \ldots, d-1\}$, the $Q F T$ is defined as follows:

$$
Q F T|v\rangle=\frac{1}{\sqrt{d}} \sum_{c=0}^{d-1} e^{2 \pi i \frac{v}{d} c}|c\rangle .
$$




\section{Our contribution}

In this section, we propose a ( $\mathrm{t}, \mathrm{n})$ threshold QSMS protocol. Let the dealers $A$ and $B$ have two secrets (for simplicity, we only take two secrets but the secrets can be any number $n$ or more than $n$, where $n$ denotes total no of players) $X$ and $Y$, respectively, and $n$ players want to jointly perform the summation $(S=X+Y)$ without revealing their secrets. In this protocol, each qualified subset $\mathbb{P}=\left\{P_{1}, P_{2}, \ldots, P_{t}\right\}$ contains a $k^{\text {th }}$ player as an initiator. We assume that $k^{\text {th }}$ player is $P_{1}$, which acts as an initiator. The initiator $P_{1}$ only contains his share value, nothing else. The process of quantum secure multiparty summation is given as follows.

Step 1: $A$ and $B$ choose two distinct $(t-1)$-degree polynomials $f(x)=X+\alpha_{1} x+\alpha_{2} x^{2}+\cdots+\alpha_{t-1} x^{t-1}$ and $g(x)=Y+\beta_{1} x+\beta_{2} x^{2}+\cdots+\beta_{t-1} x^{t-1}, X$ and $Y$ are secrets and the symbol ${ }^{\prime}{ }^{\prime}$ is defined as addition modulo $d, d$ is a prime such that $n \leq d \leq 2 n$. The $A$ and $B$ use the Shamir's secret sharing to compute the shares $f\left(x_{i}\right)$ and $g\left(x_{i}\right)$, respectively, which are distributed among $n$ players using an authenticated classical channel. The player $P_{i}$ only knows the shares $f\left(x_{i}\right)$ and $g\left(x_{i}\right), i=1,2, \ldots, n$.

Step 2: Player $P_{i}$ computes $h\left(x_{i}\right)=f\left(x_{i}\right)+g\left(x_{i}\right), i=1,2, \ldots, n$, and possesses the share $h\left(x_{i}\right)$ only.

Step 3: Player $P_{u}$ computes the shadow $\left(m_{u}\right)$ of the share $h\left(x_{u}\right), u=1,2, \ldots, t$, as follows.

$$
m_{u}=h\left(x_{u}\right) \prod_{1 \leq z \leq t, z \neq u} \frac{x_{z}}{x_{z}-x_{u}} \bmod d
$$

Step 4: Initiator player $P_{1}$ prepares $t$-particle entangled states as follows.

$$
\left|\Psi_{1}\right\rangle=\frac{1}{\sqrt{d}} \sum_{c=0}^{d-1}|c\rangle_{1}|c\rangle_{2} \ldots|c\rangle_{t}
$$

Player $P_{1}$ sends the particle $|c\rangle_{u}$ to player $P_{u}, u=2,3, \ldots, t$.

Step 5: Each player $P_{u}$ performs the $Q F T^{52}$ on his particle $|c\rangle_{u}$ as follows:

$$
Q F T|c\rangle_{1}=\frac{1}{\sqrt{d}} \sum_{a_{1}=0}^{d-1} e^{2 \pi i \frac{c}{d} a_{1}}\left|a_{1}\right\rangle .
$$

Each player $P_{u},(u=1,2, \ldots, t)$, also applies the Pauli operator $U_{m_{u}, 0}$ on his particle as follows:

$$
U_{m_{1}, 0}=\sum_{c=0}^{d-1} \omega^{c .0}\left|c+m_{1}\right\rangle\langle c|
$$

After performing the $Q F T$ and Pauli operator, the resultant state $\left|\Psi_{2}\right\rangle$ is obtained as follows.

$$
\begin{aligned}
\left|\Psi_{2}\right\rangle & =U_{m_{1}, 0} Q F T \otimes U_{m_{2}, 0} Q F T \otimes \cdots \otimes U_{m_{t}, 0} Q F T\left|\Psi_{1}\right\rangle \\
& =d^{-\frac{t+1}{2}} \sum_{0 \leq a_{1}, \ldots, a_{t}<d, a_{1}+, \ldots,+a_{t}=0}\left|a_{1}+m_{1}\right\rangle\left|a_{2}+m_{2}\right\rangle \ldots\left|a_{u}+m_{u}\right\rangle
\end{aligned}
$$

Step 6: Each player $P_{u}$ performs the measurement operation on his particle $\left|a_{u}+m_{u}\right\rangle$ in computational basis $\{|1\rangle,|2\rangle, \ldots,|d-1\rangle\}$, and broadcasts his measurement results $a_{u}+m_{u}$, where $u=1,2, \ldots, t$.

Step 7: Finally, the players in qualified subset calculate the summation jointly by summing their results of measurement: $S=\sum_{u=1}^{t} a_{u}+m_{u} \bmod d$.

\section{Correctness}

Lemma 1 If QFT and Pauli operators are honestly performed by all players in a qualified subset $\mathbb{P}=\left\{P_{1}, P_{2}, \ldots, P_{t}\right\}$, then they can jointly compute the multiparty quantum summation $\left(\sum_{u=1}^{t} m_{u} \bmod d\right)$ correctly.

Proof If QFT and Pauli operators are honestly performed by every player in the qualified subset $\mathbb{P}=\left\{P_{1}, P_{2}, \ldots, P_{t}\right\}$, the quantum state is obtained as follows. 


\begin{tabular}{|l|l|l|l|l|l|l|l|l|}
\hline \multicolumn{2}{|l|}{ Players } & $\boldsymbol{P}_{\mathbf{1}}$ & $\boldsymbol{P}_{\mathbf{2}}$ & $\boldsymbol{P}_{\mathbf{3}}$ & $\boldsymbol{P}_{\mathbf{4}}$ & $\boldsymbol{P}_{\mathbf{5}}$ & $\boldsymbol{P}_{\mathbf{6}}$ & $\boldsymbol{P}_{\mathbf{7}}$ \\
\hline \multirow{4}{*}{ Shares } & $f\left(x_{i}\right)$ & 4 & 8 & 3 & 0 & 10 & 0 & 3 \\
\cline { 2 - 9 } & $g\left(x_{i}\right)$ & 5 & 9 & 4 & 1 & 0 & 1 & 4 \\
\cline { 2 - 9 } & $h\left(x_{i}\right)$ & 9 & 6 & 7 & 1 & 10 & 1 & 7 \\
\hline
\end{tabular}

Table 1. Share computation.

$$
\begin{aligned}
\left|\Psi_{2}\right\rangle & =U_{m_{1}, 0} Q F T \otimes \cdots \otimes U_{m_{t}, 0} Q F T\left(\frac{1}{\sqrt{d}} \sum_{c=0}^{d-1}|c\rangle_{1} \ldots|c\rangle_{t}\right) \\
& =\frac{1}{\sqrt{d}} \sum_{c=0}^{d-1} U_{m_{1}, 0} Q F T|c\rangle_{1} \otimes \cdots \otimes U_{m_{t}, 0} Q F T|c\rangle_{t} \\
& =\frac{1}{\sqrt{d}} \sum_{c=0}^{d-1}\left(U_{m_{1}, 0} \frac{1}{\sqrt{d}} \sum_{a_{1}=0}^{d-1} \omega^{a_{1} c}\left|a_{1}\right\rangle\right) \otimes \cdots \otimes\left(U_{m_{t}, 0} \frac{1}{\sqrt{d}} \sum_{a_{1}=0}^{d-1} \omega^{a_{t} c}\left|a_{t}\right\rangle\right) \\
& =d^{-\frac{t+1}{2}} \sum_{0 \leq a_{1}, \ldots, a_{t}<d} \sum_{c=0}^{d-1} \omega^{\left(a_{1}+\cdots+a_{t}\right) c}\left|a_{1}+m_{1}\right\rangle \otimes \cdots \otimes\left|a_{t}+m_{t}\right\rangle \\
& =d^{-\frac{t+1}{2}} \sum_{s_{0} d} \quad\left|a_{1}+m_{1}\right\rangle \otimes \cdots \otimes\left|a_{t}+\ldots, a_{t}<d, a_{1}+\cdots+a_{t}\right\rangle=0 \bmod d
\end{aligned}
$$

Each player $P_{u}, u=1,2, \ldots, t$, performs the measurement operation on his own particle in computational basis $\left|a_{u}+m_{u}\right\rangle$. The QSMS can be computed after receiving the measurement results of each player $P_{u}$, $u=1,2, \ldots, t$. The QSMS of secret can be calculated as follows.

$$
\sum_{u=1}^{t} a_{u}+m_{u} \stackrel{d}{=} \sum_{u=1}^{t} a_{u}+\sum_{u=1}^{t} m_{u} \stackrel{d}{=} \sum_{u=1}^{t} m_{u} \bmod d
$$

Thus, the multiparty quantum summation of secrets equals to $\sum_{u=1}^{t} m_{u} \bmod d$.

\section{Illustration of secure multiparty quantum summation}

Here, we use a numerical example to discuss the working of the proposed protocol. Let $A$ and $B$ hold two secrets 2 and 3 , respectively and they want to perform the summation $S=(2+3)$. $A$ and $B$ choose threshold $(t)=3$, total number of players $(n)=7$, and prime $(d)=11$. Suppose $A$ and $B$ select two different polynomials $f(x)=2+x+x^{2}$ mod 11 and $g(x)=3+x+x^{2}$ mod 11, respectively. They calculate the shares $f\left(x_{i}\right)$ and $g\left(x_{i}\right), i=1,2, \ldots, 7$ using the Shamir's secret sharing, and allocate these shares to 7 players. Each player $P_{i}, i=1,2, \ldots, 7$, performs $h\left(x_{i}\right)=f\left(x_{i}\right)+g\left(x_{i}\right)$ mod 11 . The calculation of shares $h\left(x_{i}\right)$ is shown in Table 1. Each player $P_{u}, \mathrm{u}=1,2,3$, computes the shadow of the shares $m_{u}$, as $m_{1}=9 \cdot\left(\frac{2}{2-1} \cdot \frac{3}{3-1}\right) \bmod 11=5$, $m_{2}=6 \cdot\left(\frac{1}{1-2} \cdot \frac{3}{3-2}\right) \bmod 11=4$, and $m_{3}=7 \cdot\left(\frac{1}{1-3} \cdot \frac{2}{2-3}\right) \quad \bmod 11=7$, respectively (using Eq. 5 ). The player $P_{1}$ now computes $\left|\Psi_{1}\right\rangle=\frac{1}{\sqrt{11}} \sum_{c=0}^{10}|c\rangle_{1}|c\rangle_{2}|c\rangle_{3}$ and sends the particle $|c\rangle_{u}$ to player $P_{u}, u=2,3$. Each player $P_{u}, u=1,2,3$, applies the QFT and Pauli operator $U_{5,0}, U_{4,0}, U_{7,0}$ on his particle, respectively, (as per Eq. 9).

$$
\begin{aligned}
\left|\Psi_{2}\right\rangle & =U_{5,0} Q F T \otimes U_{4,0} Q F T \otimes U_{7,0} Q F T\left(\frac{1}{\sqrt{11}} \sum_{c=0}^{10}|c\rangle_{1}|c\rangle_{2}|c\rangle_{3}\right) \\
& =\frac{1}{\sqrt{11}} \sum_{c=0}^{10} U_{5,0} Q F T|c\rangle_{1} \otimes U_{4,0} Q F T|c\rangle_{2} \otimes U_{7,0} Q F T|c\rangle_{3} \\
& =11 r_{1} \sum_{0 \leq a_{1}, a_{2}, a_{3}<10, a_{1}+a_{2}+a_{3}=0} \bmod 11
\end{aligned}
$$

Each player $P_{u}, u=1,2,3$, performs the measurement operation in computational basis on his particle. The players $P_{1}, P_{2}$, and $P_{3}$ broadcast the measurement results $a_{1}+5, a_{2}+4$, and $a_{3}+7$, respectively. Finally, they get the summation by summing the results of measurement as follows:

$$
a_{1}+5+a_{2}+4+a_{3}+7 \stackrel{11}{\equiv} a_{1}+a_{2}+a_{3}+16 \stackrel{11}{\equiv} 16 \quad \bmod 11=5 .
$$




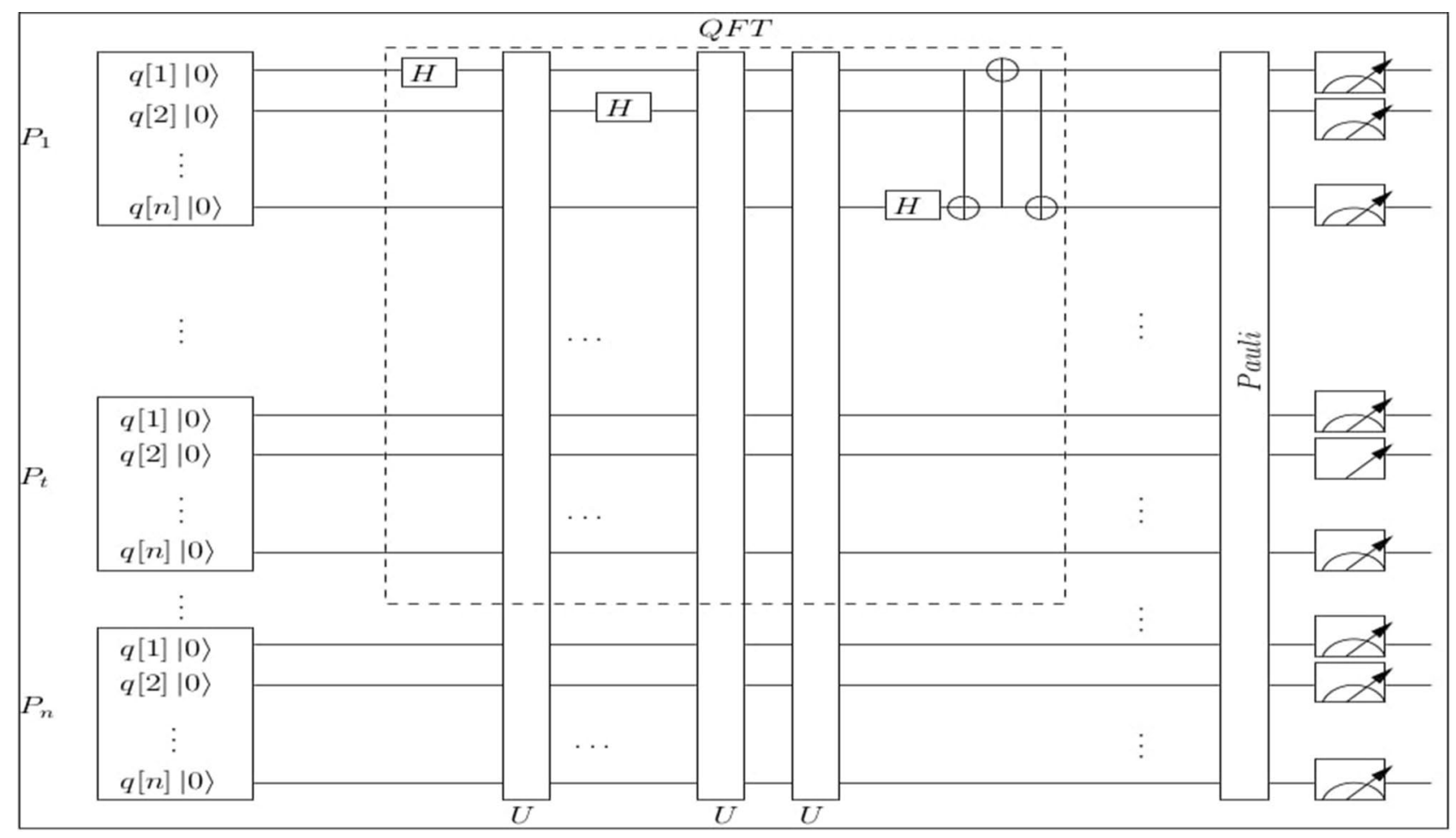

Figure 1. Circuit diagram of QSMS.

\section{Simulation results}

We simulate the proposed protocol using the IBM real quantum processor ${ }^{39,40}$, which is available at T.J.Watson lab, USA. We explain the circuit diagram (refer Fig. 1) of our QSMS protocol. The Hadamard gate is taken as the QFT in this circuit diagram of QSMS. On his particle, the player $P_{u}$ applies the QFT and also performs the Pauli operator on his particle. Then, each player $P_{u}$ performs measurement operations on his own particle, and broadcasts the measurement result. Finally, by summing their measurement results, the players jointly calculate the QSMS. The privacy of this protocol is guaranteed until a certain number of players disclose their shares.

We have simulated this circuit of QSMS with 3 players, 5 qubits, and 8192 number of average shots. Initially, the player $P_{u}, u=1,2,3$ performs the $Q F T$ on his particle $|c\rangle_{u}$ and also executes the Pauli operator on particle $|c\rangle_{u}$. Then, each player $P_{u}, u=1,2,3$, executes the measurement operation in computational basis on his particle. The players $P_{1}, P_{2}$, and $P_{3}$ broadcast the measurement results $a_{1}+5, a_{2}+4$, and $a_{3}+7$, respectively. Finally, they get the summation of 2 and 3 by adding the measurement results as follows:

$$
a_{1}+5+a_{2}+4+a_{3}+7=16 \bmod 11=5 .
$$

The simulation result of the proposed summation protocol for 3 players, 5 qubits, and 8192 number of average shots. The state 101 (i.e., binary representation of 5) is calculated efficiently. The result of this simulation using the IBM real quantum processor is shown in Fig. 2.

\section{Discussion}

Here, we address the security and performance analysis based on some properties of the proposed QSMS protocol.

Security analysis. In this section, we analyze the security of QSMS protocol based on the intercept-resend, entangle-measure, intercept, collective, coherent, and collusion attacks.

Intercept-resend attack Suppose an attacker Mallory intercepts the particle $|c\rangle_{u}$. It measures the quantum particle $|c\rangle_{u}$ in the computational basis to get the useful data about the share's shadow $\left(m_{u}\right)$. Mallory produces the clone quantum particle $|\bar{c}\rangle_{u}$ and resends this clone particle to player $P_{u}, u=2,3, \ldots t$. If Mallory applies this method to attack, then it can get $c$ accurately with probability $\frac{1}{d}$. But, from this attack, Mallory cannot get any useful data about the share's shadow $m_{u}$, because the intercepted particle $|c\rangle_{u}$ does not contain any useful data about the share's shadow $m_{u}$.

Entangle-Measure attack After the intercept attack, Mallory performs the complex entangle-measure attack on the entangled quantum particle $|c\rangle_{u}$. In this attack, Mallory performs the measurement operation on the intercepted entangled quantum particle $|c\rangle_{u}$ in the computational basis to get the useful data about the share's shadow $m_{u}$. If Mallory applies the entangle-measure attack, then it can get $c$ accurately with probability $\frac{1}{d}$. But, from this attack, Mallory cannot get useful data about the share's shadow $m_{u}$, because the intercepted entangled quantum particle $|c\rangle_{u}$ does not contain any useful data about the share's shadow $m_{u}$. 


\section{Quantum State: Computation Basis}

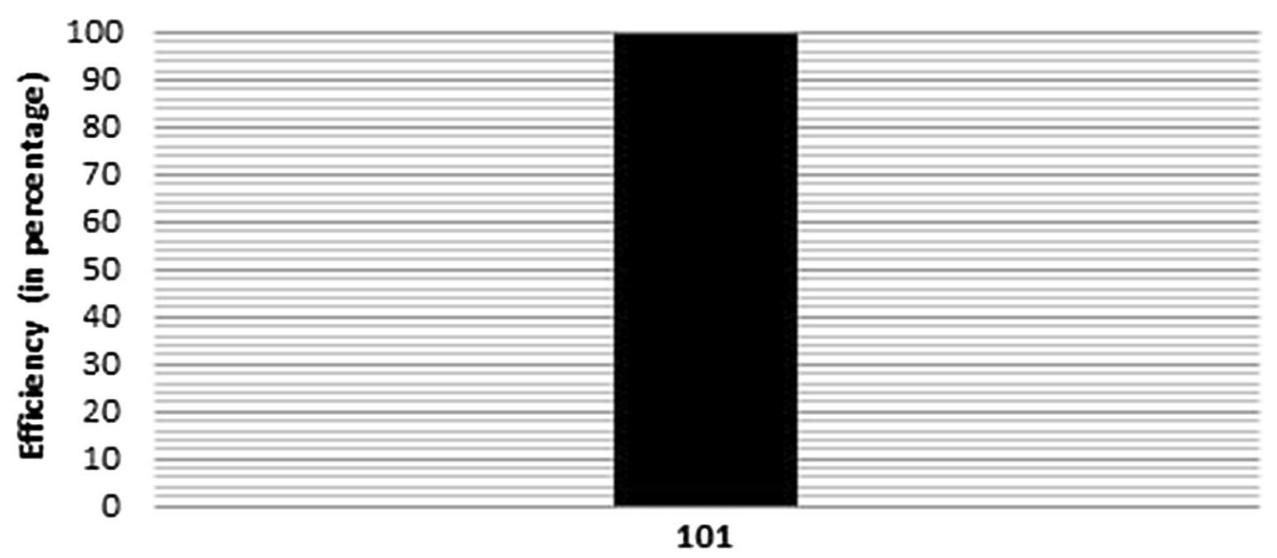

Figure 2. Simulation result.

Intercept attack Suppose Mallory intercepts the particle $|c\rangle_{u}$ and measures the quantum particle $|c\rangle_{u}$ in the computational basis to reveal the useful data about the share's shadow $m_{u}$. If Mallory measures the quantum particle $|c\rangle_{u}$ in the computational basis, then it can get $c$ correctly with probability $\frac{1}{d}$. But, from the measurement result $c$, it cannot get any useful data about the share's shadow $m_{u}$, because the intercepted particle $|c\rangle_{u}$ does not carry any useful data about the share's shadow $m_{u}$.

Collective attack In a collective attack, Mallory prepares an autonomous ancillary particle to communicate with each qudit to get the shadow of share and they perform the joint measurement operation on every ancillary qudit. Suppose Mallory communicates with every qudit of all players by preparing an autonomous ancillary particle $|e\rangle$. After successful interaction, Mallory gets the particle $|o\rangle_{x}$. Then, Mallory wants to know the shadow of share by performing a computational basis $\{|1\rangle,|2\rangle, \ldots,|d-1\rangle\}$ joint measurement operation. Mallory cannot get any useful data about the share's shadow from this joint measurement operation because $|o\rangle_{x}$ does not contain any useful data about the share's shadow.

Coherent attack In this attack, Mallory prepares an autonomous ancillary particle $|c\rangle$ to communicate with the qudits of each player. After interacting, Mallory gets each player's particle $|o\rangle_{x}$ and performs a joint measurement operation on all players particle $c$ in computational basis $\{|1\rangle,|2\rangle, \ldots,|d-1\rangle\}$. Mallory only gets $o$ from the joint measurement result of particle $|o\rangle_{x}$ with probability $\frac{1}{d}$. But, the joint measurement result $o$ does not contain any useful data about the share's shadow. From this attack, Mallory only gets the interacting particle $|o\rangle_{x}$, but it cannot learn any useful data about the share's shadow.

Collusion attack In this protocol, each player $P_{u}$ performs the measurement on his own particle $\left|a_{u}+m_{u}\right\rangle$ and broadcasts his result of the measurement $a_{u}+m_{u}, u=1,2, \ldots, t$. From this broadcast, other players cannot get any useful data about the share's shadow $m_{u}$. If some rational players $P_{l-1}$ and $P_{l+1}$ jointly want to get the data about the share's shadow but they cannot get any useful data about the share's shadow $m_{u}$ because the initiator $P_{1}$ transmits only particles $|c\rangle_{u}$ to all other players and unfortunately $|c\rangle_{u}$ does not contain any useful data about the share's shadow $m_{u}$.

Performance analysis. We analyze and compare the performance of the proposed $(t, n)$ threshold summation protocol with the existing summation protocols $s^{44-54}$. The protocols ${ }^{44-47}$ are multiparty, but they have the threshold approach of $(n, n)$ and their type of computation is bit-by-bit. The protocol ${ }^{48}$ is multiparty and its type of computation is secret-by-secret, but it is based on the threshold approach of $(n, n)$. The protocols ${ }^{49,50}$ perform bit-by-bit computation, but they are based on the threshold approach of $(n, n)$. The protocol ${ }^{51}$ is multiparty, but its type of computation is bit-by-bit and it has the threshold approach of $(n, n)$ with modulo is 2 . The protocol ${ }^{52}$ is multiparty and its type of computation is secret-by-secret, but it is based on the threshold approach of $(n, n)$. The protocol $l^{53}$ is based on quantum multiparty computation, but its type of computation is bit-by-bit and it has the threshold approach of $(n, n)$. The protocol ${ }^{54}$ is multiparty and its type of computation is secret-by-secret, but it has the threshold approach of $(n, n)$, where all honest players need to perform the multiparty quantum summation. This protocol cannot be performed correctly if any player is dishonest. However, our proposed protocol has the threshold approach of $(t, n)$, in which only honest players of $t$ can securely compute the multiparty quantum summation with modulo $d$. In addition, the proposed protocol has secret-by-secret computation type. This protocol can be performed correctly if any $t$ players are honest. So, Compared to other protocols, our proposed protocol is more cost-effective, efficient, realistic, and secure, as shown in Table 2. In this table, Com., Comm., UO, Part., MO, QFT, QFT ${ }^{-1}, D P, E M, I N C P T, M P, C O L L, C O L, C O H, I R$, sec - by - sec, Y, N, MD, and CT denote Computation, Communication, Unitary Operation, Participant, Measure Operation, Quantum Fourier Transform, Inverse Quantum Fourier Transform, Decoy Particle, Entangle-Measure, Intercept, Message Particle, Collective, Collusion, Coherent, Intercept-Resend, secret-by-secret, Yes, No, Modulo, and type of Computation, respectively. 


\begin{tabular}{|c|c|c|c|c|c|c|c|c|c|c|c|c|c|c|c|c|}
\hline \multirow[b]{4}{*}{ Protocols } & \multicolumn{16}{|c|}{ Performance parameters } \\
\hline & \multicolumn{6}{|c|}{ Costs } & \multicolumn{6}{|c|}{ Attacks } & \multicolumn{4}{|c|}{ Universality } \\
\hline & \multicolumn{4}{|l|}{ Com. } & \multicolumn{2}{|c|}{ Comm. } & \multicolumn{3}{|c|}{ Outside } & \multicolumn{3}{|l|}{ Part. } & \multirow[b]{2}{*}{ Model } & \multirow[b]{2}{*}{ MD } & \multirow[b]{2}{*}{ Qubit } & \multirow[b]{2}{*}{ CT } \\
\hline & QFT & $Q F T^{-1}$ & MO & UO & $M P$ & $D P$ & $I R$ & $E M$ & INCPT & COLL & $\mathrm{COH}$ & $\mathrm{COL}$ & & & & \\
\hline Ref. ${ }^{44}$ & - & - & - & - & - & - & - & - & $N$ & - & - & - & $(n, n)$ & $n+1$ & - & Bit-by-bit \\
\hline Ref. $^{45}$ & - & - & - & 1 & - & - & $Y$ & - & $N$ & - & - & $Y$ & $(n, n)$ & 2 & - & Bit-by-bit \\
\hline Ref. $^{46}$ & - & - & - & 1 & - & - & $Y$ & $Y$ & $N$ & - & - & $N$ & $(n, n)$ & 2 & - & Bit-by-bit \\
\hline Ref. $^{47}$ & - & - & - & 1 & - & - & $Y$ & - & $N$ & - & - & $N$ & $(n, n)$ & 2 & - & Bit-by-bit \\
\hline Ref. $^{48}$ & 1 & 1 & 2 & $n-1$ & - & $n$ & $Y$ & $Y$ & $N$ & - & - & - & $(n, n)$ & $p$ & $\left\lceil\log _{2}^{d}\right\rceil n$ & $\begin{array}{l}\text { Sec-by- } \\
\text { sec }\end{array}$ \\
\hline Ref. $^{49}$ & - & - & - & - & - & - & - & - & $N$ & - & - & - & $(n, n)$ & - & $2\left\lceil\log _{2}^{d}\right\rceil n$ & Bit-by-bit \\
\hline Ref. $^{50}$ & - & - & $n$ & - & - & - & $Y$ & - & $N$ & - & - & $Y$ & $(n, n)$ & 2 & - & Bit-by-bit \\
\hline Ref. $^{51}$ & - & - & $n$ & - & - & $n$ & $Y$ & $Y$ & $N$ & - & - & - & $(n, n)$ & 2 & 2 & Bit-by-bit \\
\hline Ref. $^{52}$ & $n$ & - & $n$ & - & - & $n-1$ & $Y$ & $Y$ & $N$ & - & - & $Y$ & $(n, n)$ & $d$ & - & $\begin{array}{l}\text { Sec-by- } \\
\text { sec }\end{array}$ \\
\hline Ref. $^{53}$ & - & - & $n$ & $n$ & - & $n$ & $Y$ & $Y$ & $N$ & - & - & - & $(n, n)$ & $p$ & - & Bit-by-bit \\
\hline Ref. $^{54}$ & - & - & $n$ & 1 & - & - & $Y$ & $Y$ & $N$ & - & - & $Y$ & $(n, n)$ & - & - & $\begin{array}{l}\text { Sec-by- } \\
\text { sec }\end{array}$ \\
\hline Proposed & 1 & - & $t$ & $t-1$ & $t-1$ & - & $Y$ & $Y$ & $Y$ & Y & Y & $Y$ & $(t, n)$ & $d$ & - & $\begin{array}{l}\text { Sec-by- } \\
\text { sec }\end{array}$ \\
\hline
\end{tabular}

Table 2. Comparison with ten protocols.

\section{Conclusion}

In this paper, we have discussed a secret sharing based $(t, n)$ threshold QSMS protocol. This protocol can be executed efficiently if any $t$ number of players are honest. It is secure and efficient because its type of computation is secret-by-secret and its communication type is linear. It can also compute the QSMS if the total number of secrets is more than the total number of players because the linear secret sharing is used to compute the share of secrets. This QSMS protocol is more realistic as compared to the existing multiparty quantum summation protocols because we have simulated this protocol efficiently using IBM quantum computer that provides efficient result after increasing the number of shots.

Received: 15 September 2020; Accepted: 23 December 2020

Published online: 26 January 2021

\section{References}

1. Gyongyosi, L. \& Imre, S. A survey on quantum computing technology. Comput. Sci. Rev. 31, 51-71 (2019).

2. Gyöngyösi, L., Bacsardi, L. \& Imre, S. A survey on quantum key distribution. Infocommun. J. 11, 14-21 (2019).

3. Gyongyosi, L. Multicarrier continuous-variable quantum key distribution. Theor. Comput. Sci. 816, 67-95 (2020).

4. Braunstein, S. L. \& Pirandola, S. Side-channel-free quantum key distribution. Phys. Rev. Lett. 108, 130502 (2012).

5. Ghalaii, M., Ottaviani, C., Kumar, R., Pirandola, S. \& Razavi, M. Discrete-modulation continuous-variable quantum key distribution enhanced by quantum scissors. IEEE J. Sel. Areas Commun. 38, 506-516 (2020).

6. Gyongyosi, L., Imre, S. \& Nguyen, H. V. A survey on quantum channel capacities. IEEE Commun. Surv. Tutor. 20, 1149-1205 (2018).

7. Gyongyosi, L. \& Imre, S. Circuit depth reduction for gate-model quantum computers. Sci. Rep. 10, 1-17 (2020).

8. Gyongyosi, L. \& Imre, S. Quantum circuit design for objective function maximization in gate-model quantum computers. Quant. Inf. Process. 18, 225 (2019).

9. Pirandola, S., Laurenza, R., Ottaviani, C. \& Banchi, L. Fundamental limits of repeaterless quantum communications. Nat. Commun. 8, 1-15 (2017).

10. Gyongyosi, L. \& Imre, S. Optimizing high-efficiency quantum memory with quantum machine learning for near-term quantum devices. Sci. Rep. 10, 1-24 (2020).

11. Gyongyosi, L. \& Imre, S. Dense quantum measurement theory. Sci. Rep. 9, 1-18 (2019).

12. Van Meter, R. Quantum Networking (Wiley, Hoboken, 2014).

13. Laurenza, R., Lupo, C., Lloyd, S. \& Pirandola, S. Dense coding capacity of a quantum channel. Phys. Rev. Res. 2, 023023 (2020).

14. Wang, B., Sun, Y., Duong, T. Q., Nguyen, L. D. \& Zhao, N. Popular matching for security-enhanced resource allocation in social internet of flying things. IEEE Trans. Commun. (2020).

15. Sun, Z. et al. Towards practical quantum secure direct communication: A quantum-memory-free protocol and code design. IEEE Trans. Commun. (2020).

16. Yuan, R. \& Cheng, J. Free-space optical quantum communications in turbulent channels with receiver diversity. IEEE Trans. Commun. (2020).

17. Pirandola, S. Quantum discord as a resource for quantum cryptography. Sci. Rep. 4, 6956 (2014).

18. Pirandola, S. End-to-end capacities of a quantum communication network. Commun. Phys. 2, 1-10 (2019).

19. Pirandola, S. Limits and security of free-space quantum communications. arXiv:2010.04168 (2020).

20. Karafyllidis, I. G. Quantum computer simulator based on the circuit model of quantum computation. IEEE Trans. Circuit. Syst. Regul. Pap. 52, 1590-1596 (2005).

21. Ju, Y.-L., Tsai, I.-M. \& Kuo, S.-Y. Quantum circuit design and analysis for database search applications. IEEE Trans. Circuit. Syst. Regul. Pap. 54, 2552-2563 (2007). 
22. Li, H.-S., Fan, P., Xia, H.-Y., Peng, H. \& Song, S. Quantum implementation circuits of quantum signal representation and type conversion. IEEE Trans. Circuit. Syst. Regul. Pap. 66, 341-354 (2018).

23. Guo, W. \& Oruç, A. Y. Regular sparse crossbar concentrators. IEEE Trans. Comput. 47, 363-368 (1998).

24. Sue, C.-C. An enhanced universal $\mathrm{n} \mathrm{x}$ fully nonblocking quantum switch. IEEE Trans. Comput. 58, 238-250 (2008).

25. Lu, Y., Shu, J., Guo, J., Li, S. \& Mutlu, O. High-performance and lightweight transaction support in flash-based ssds. IEEE Trans. Comput. 64, 2819-2832 (2015).

26. Aswal, A., Perumal, G. \& Prasanna, G. S. P. On basic financial decimal operations on binary machines. IEEE Trans. Comput. 61, 1084-1096 (2012).

27. Turcu, A., Palmieri, R. \& Ravindran, B. On open nesting in distributed transactional memory. IEEE Trans. Comput. 65, 1856-1868 (2015).

28. Bui, B. D., Pellizzoni, R. \& Caccamo, M. Real-time scheduling of concurrent transactions in multidomain ring buses. IEEE Trans. Comput. 61, 1311-1324 (2011).

29. Wang, J.-Y. et al. Direct and full-scale experimental verifications towards ground-satellite quantum key distribution. Nat. Photon. 7, 387 (2013).

30. Lo, H.-K., Curty, M. \& Tamaki, K. Secure quantum key distribution. Nat. Photon. 8, 595 (2014).

31. Xu, F., Curty, M., Qi, B., Qian, L. \& Lo, H.-K. Discrete and continuous variables for measurement-device-independent quantum cryptography. Nat. Photon. 9, 772-773 (2015).

32. Cheng, S.-T. \& Wang, C.-Y. Quantum switching and quantum merge sorting. IEEE Trans. Circuit. Syst. Regul. Pap. 53, 316-325 (2006).

33. Kong, B. Y., Yoo, H. \& Park, I.-C. Efficient sorting architecture for successive-cancellation-list decoding of polar codes. IEEE Trans. Circuit. Syst. Express Briefs 63, 673-677 (2016).

34. Nakamura, S. \& Masson, G. M. Lower bounds on crosspoints in concentrators. IEEE Trans. Comput. 1, 1173-1179 (1982).

35. Lydersen, L. et al. Hacking commercial quantum cryptography systems by tailored bright illumination. Nat. Photon. 4, 686 (2010).

36. Shu, H., Yu, R., Jiang, W. \& Yang, W. Efficient implementation of $k$-nearest neighbor classifier using vote count circuit. IEEE Trans. Circuit. Syst. Express Briefs 61, 448-452 (2014).

37. Gisin, N. \& Thew, R. Quantum communication. Nat. Photon. 1, 165 (2007).

38. Shamir, A. How to share a secret. Commun. ACM 22, 612-613 (1979).

39. Castelvecchi, D. Ibm's quantum cloud computer goes commercial. Nat. News 543, 159 (2017).

40. Sisodia, M., Shukla, A. \& Pathak, A. Experimental realization of nondestructive discrimination of bell states using a five-qubit quantum computer. Phys. Lett. A 381, 3860-3874 (2017).

41. Heinrich, S. Quantum summation with an application to integration. J. Compl. 18, 1-50 (2002).

42. Heinrich, S., Kwas, M. \& Woźniakowski, H. Quantum boolean summation with repetitions in the worst-average setting. in Monte Carlo and Quasi-Monte Carlo Methods 2002, 243-258 (Springer, New York, 2004).

43. Hillery, M., Ziman, M., Bužek, V. \& Bieliková, M. Towards quantum-based privacy and voting. Phys. Lett. A 349, 75-81 (2006).

44. Jian-Zhong, C., Xiu-Bo, Du. ann \& Qiao-Yan, W. Secure multiparty quantum summation. Acta Phys. Sin. 56, 6214-6219 (2007).

45. Chen, X.-B., Xu, G., Yang, Y.-X. \& Wen, Q.-Y. An efficient protocol for the secure multi-party quantum summation. Int. J. Theor. Phys. 49, 2793-2804 (2010).

46. Zhang, C., Sun, Z., Huang, Y. \& Long, D. High-capacity quantum summation with single photons in both polarization and spatialmode degrees of freedom. Int. J. Theor. Phys. 53, 933-941 (2014).

47. Zhang, C., Sun, Z.-W., Huang, X. \& Long, D.-Y. Three-party quantum summation without a trusted third party. Int. J. Quant. Inf. 13, 1550011 (2015).

48. Shi, R.-H., Mu, Y., Zhong, H., Cui, J. \& Zhang, S. Secure multiparty quantum computation for summation and multiplication. Sci. Rep. 6, 19655 (2016).

49. Shi, R.-H. \& Zhang, S. Quantum solution to a class of two-party private summation problems. Quant. Inf. Process. 16, 225 (2017).

50. Zhang, C., Situ, H., Huang, Q. \& Yang, P. Multi-party quantum summation without a trusted third party based on single particles. Int. J. Quant. Inf. 15, 1750010 (2017).

51. Liu, W., Wang, Y.-B. \& Fan, W.-Q. An novel protocol for the quantum secure multi-party summation based on two-particle bell states. Int. J. Theor. Phys. 56, 2783-2791 (2017).

52. Yang, H.-Y. \& Ye, T.-Y. Secure multi-party quantum summation based on quantum fourier transform. Quant. Inf. Process. 17, 129 (2018).

53. Lv, S.-X., Jiao, X.-F. \& Zhou, P. Multiparty quantum computation for summation and multiplication with mutually unbiased bases. Int. J. Theor. Phys. 58, 2872-2882 (2019).

54. Zhang, C., Razavi, M., Sun, Z. \& Situ, H. Improvements on "secure multi-party quantum summation based on quantum fourier transform". Quant. Inf. Process. 18, 336 (2019).

55. Sutradhar, K. \& Om, H. Efficient quantum secret sharing without a trusted player. Quant. Inf. Process. 19, 73 (2020).

56. Sutradhar, K. \& Om, H. A generalized quantum protocol for secure multiparty summation. IEEE Trans. Circuits Syst. II (2020).

57. Sutradhar, K. \& Om, H. Hybrid quantum protocols for secure multiparty summation and multiplication. Sci. Rep. 10, 1-9 (2020).

58. Nielsen, M. A. \& Chuang, I. Quantum Computation and Quantum Information (Springer, New York, 2002).

\section{Acknowledgements}

This work is partially supported by Indian Institute of technology (ISM) Dhanbad.

\section{Author contributions}

Study conception, design, and writing of the manuscript: K.S. Analysis: H.O. All authors reviewed the manuscript.

\section{Competing interests}

The authors declare no competing interests.

\section{Additional information}

Correspondence and requests for materials should be addressed to K.S.

Reprints and permissions information is available at www.nature.com/reprints.

Publisher's note Springer Nature remains neutral with regard to jurisdictional claims in published maps and institutional affiliations. 
(c) (i) Open Access This article is licensed under a Creative Commons Attribution 4.0 International cc) License, which permits use, sharing, adaptation, distribution and reproduction in any medium or format, as long as you give appropriate credit to the original author(s) and the source, provide a link to the Creative Commons licence, and indicate if changes were made. The images or other third party material in this article are included in the article's Creative Commons licence, unless indicated otherwise in a credit line to the material. If material is not included in the article's Creative Commons licence and your intended use is not permitted by statutory regulation or exceeds the permitted use, you will need to obtain permission directly from the copyright holder. To view a copy of this licence, visit http://creativecommons.org/licenses/by/4.0/.

(C) The Author(s) 2021 